\title{
Corrosion Damage Detection with Piezoelectric Wafer Active Sensors
}

\author{
Dustin Thomas, John Welter \\ Air Force Research Lab, Materials and Manufacturing Directorate, NDE Branch \\ Wright-Patterson AFB, OH 45433, Dustin.Thomas@wpafb.af.mil \\ Victor Giurgiutiu \\ Mechanical Engineering Department, University of South Carolina \\ Columbia, SC 29208, victorg@sc.edu
}

\begin{abstract}
Since today's aging fleet is now intended to far exceed their proposed design life, monitoring the structural integrity of those aircraft has become a priority issue for today's Air Force. One of the most critical structural problems is corrosion. In fact the $\mathrm{KC}-135$ now costs $\$ 1.2$ billion a year to repair corrosion. In this paper, we plan to show the use of Lamb waves to detect material loss in thin plates representative of aircraft skins. To do this we will use embedded transducers called Piezoelectric Wafer Active Sensor (PWAS) in a pitch-catch configuration. The sensors were placed on a grid pattern. Material loss through corrosion was simulated by removing the material mechanically with an abrasive tool. Thus, simulated corrosion pits of various depths and area coverage were made. Three-count tone burst wave packets were used. The Lamb wave packets were sent in a pitch-catch mode from one transmitter PWAS to the other PWAS in the grid acting as receivers. The Lamb wave mode used in these experiments was $A_{1}$, since this was found to be more sensitive to changes due to material loss. At the frequencies considered in our experiments, the $A_{1}$ waves are highly dispersive. It was found that, as the Lamb wave travels through simulated corrosion damage, the signal changes. The observed changes were in the signal wavelength (due to change in the dispersive properties of the medium) and in signal amplitude (due to redistribution of energy in the wave packet). This change in signal can be correlated to the magnitude of damage. To achieve this, we have used several approaches: (a) direct correlation between the sent and the received signals; (b) wavelet transform of the signal followed by correlation of the wavelet coefficients time-frequency maps; (c) Hilbert transform of the signal to produce the signal envelope and comparison of the resulting envelope signals (d) neural network correlation between the sent and received signals. It was found that these methods work well together in a complementary way.
\end{abstract}

Keywords: structural health monitoring, aircraft corrosion, active sensors, piezoelectric, aging aircraft, cracks, damage, faults, diagnostics, prognostics, PWAS, Lamb waves, NDE, pitch-catch 


\section{INTRODUCTION}

The object of our study was to detect the presence of corrosion in aluminum plates with in-situ piezoelectric wafer active sensors (PWAS). We considered a large square plate on which two 10-mm diameter PWAS were placed on a diagonal (Figure 1). Corrosion damage was simulated in the middle of the plate using an acid etching method. As shown in Figure 1, the corrosion damage was placed directly on the line connecting the two PWAS. The pitch-catch method with $\mathrm{A}_{0}$ Lamb waves was used for damage detection. The $\mathrm{A}_{0}$ Lamb waves were generated by one PWAS and detected by the other PWAS. Measurements were taken before and after applying the simulated corrosion damage. When traversing simulated corrosion regions, the $\mathrm{A}_{0}$ Lamb waves changed their characteristics. Comparison of the wave characteristics between "pristine" and "corrosion" would serve as an indicator of the corrosion presence.

The paper starts with a presentation of the state of the art in ultrasonic corrosion detection using Lamb waves. This is followed by a theoretical analysis, followed by the description of the experimental setup, results, and data processing and interpretation. The theoretical analysis presents the basis for $\mathrm{A}_{0}$ mode tuned excitation with PWAS, and gives predictions of the tuning frequency that are later confirmed by the experimental results. Also given in the theoretical section is a prediction of how the dispersive $\mathrm{A}_{0}$ Lamb waves are affected by plate thickness changes. A method for quantifying the effect of small thickness changes on the $A_{0}$ Lamb waves using cross plots between pristine and corroded signals is presented in the theoretical section using simulated data. This method is further validated in the experimental section using actual data measured during the tests.

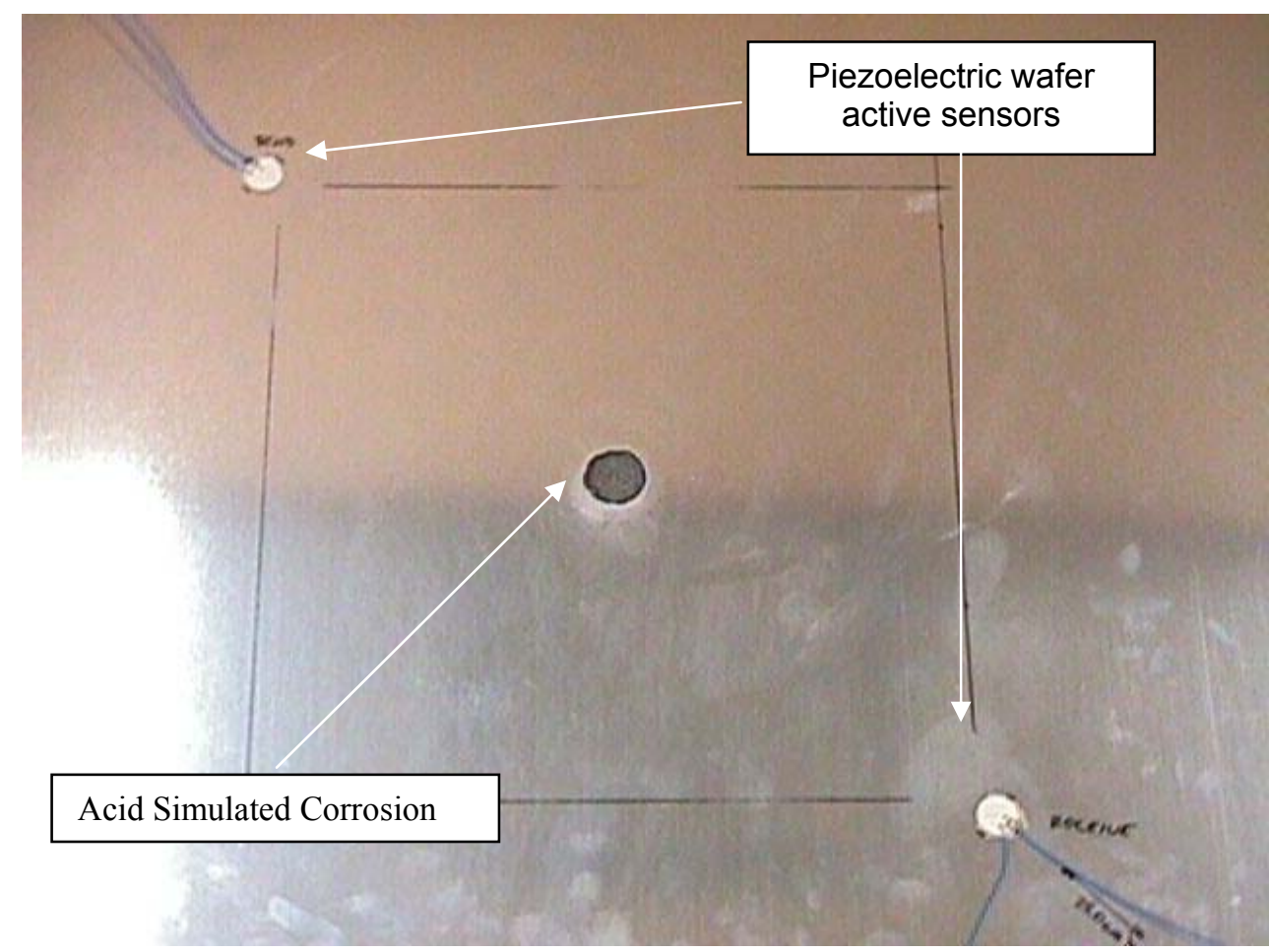

Figure 1 Corrosion detection experimental setup containing two 10-mm diameter PWAS at $250 \mathrm{~mm}$. Corrosion was simulated by acid damage between the two PWAS. 


\section{STATE OF THE ART}

Lamb waves are ultrasonic guided waves that can travel at large distance in thin wall structures and facilitate efficient detection of damage over large areas ${ }^{1,2}$. Of particular interest is the use of Lamb waves for the detection of corrosion. Chahbaz et al. ${ }^{3}$ demonstrates the ability of Lamb waves to detect corrosion through material loss. They used ultrasonic NDE transducers and wedges to induce and detect Lamb waves in aluminum test articles. This showed that Lamb wave modes could detect visible thinning as well as thinning that was hidden under patches and lap joints when the transducers were operated in a pitch-catch setup being excited in a high power narrow bandwidth mode of operation. They indicated that $\mathrm{A}_{1}$ guided Lamb waves transmitted through the corroded area result in a received signal of lower amplitude and longer time of flight than when passing through a pristine area. This is consistent with the theoretical calculations of the group velocity dispersion curves and of signal attenuation. Mode selection and optimization can improve the resolution of the material loss estimation. Experiments were performed to detect corrosion around rivets and other fasteners. Alleyne et al. ${ }^{6}$ used guided waves to detect corrosion in steel pipes based on mode conversion. Jenot et al. ${ }^{6}$ used wedges and transducers to generate symmetric $\mathrm{S}_{0}$ Lamb waves, which they sent along a copper plate. In sending the wave through a corroded area of the plate and through an uncorroded area of the plate they could detect changes in the group velocity which they was attributed to material thinning. They also showed that they were able to detect the material damage when they sent the signal along the back of the plate as well. This is of particular note since this technique could be used to detect hidden corrosion. Sicard et al. ${ }^{8}$ applied this method to corrosion detection in airframe structures.

In recent years, the use of embedded piezoelectric wafer active sensors (PWAS) for the detection of material damage with Lamb waves techniques has experienced an ascending trend. Keilers and Chang ${ }^{9}$ (1995) were among the early researchers to experiment with in-situ piezoelectrics for detection of material damage through wave propagation and demonstrated the use of this technique on a composite beam. Wang and Chang ${ }^{10}$ extended this method to delamination damage identification in a composite plate. They showed that it is possible to detect impact damage in composite structures with four piezoelectric wafer transducers operating in a pitch-catch mode. The $\mathrm{A}_{0}$ Lamb wave mode was excited and the location of the damage was determined through processing of scatter and time of flight data. Kessler et al. ${ }^{14}$ have further studied damage detection in composite structures. They have been working towards optimizing sensor placement in the structure. In their experiments, they demonstrated that Lamb wave techniques are able to show more defect information than frequency response methods since the Lamb wave techniques are more sensitive to local damage effects. Lemistre et al. ${ }^{12}$ studied the propagation of PWAS generated Lamb waves in laminated composite materials and their diffraction by defects. They showed that $\mathrm{S}_{0}$ mode Lamb waves incident onto a delaminated region are diffracted as $\mathrm{S}_{0}, \mathrm{~A}_{0}$, and $\mathrm{SH}_{0}$ guided waves through the mode conversion phenomenon. The wavelet transform decomposition was used to extract the various guided wave modes generated by the diffraction process. Giurgiutiu et al. ${ }^{13}$ has shown in his work that a single PWAS operating in the $\mathrm{S}_{0}$ symmetric Lamb wave mode at $300 \mathrm{kHz}$ could detect cracks through pulse-echo excitation and signal processing. Through utilization of several PWAS transducers in a phased array configuration, cracks and their corresponding locations could be determined and visualized ${ }^{14}$.

Examination of the state of the art has revealed that relevant previous work exists in two main directions: (a) the use of Lamb waves for corrosion detection; and (b) the use of in-situ piezoelectric sensors for damage detection using Lamb waves. However, our work consists of using embedded piezoelectric wafer active sensors (PWAS) for the corrosion detection, i.e. using the methods (a) and (b) in combination. To date, we have been unable to find any reported work on using these two methods in combination, i.e., of using embedded PWAS for corrosion detection as described in the present work. 


\section{THEORY}

Consider a uniform plate with a PWAS transducer bonded to its top surface. When the PWAS is excited by a harmonic electric voltage $V=V_{0} e^{i \omega t}$, elastic Lamb waves are excited inside the structure (Figure 2). The interaction between the PWAS and the structure takes place through the interfacial shear stress $\tau=\tau_{0}(x) e^{i \omega t}$. The exact spatial distribution of $\tau_{0}(x)$ depends on the properties of the bonding layer between the PWAS and the structure.

(a)
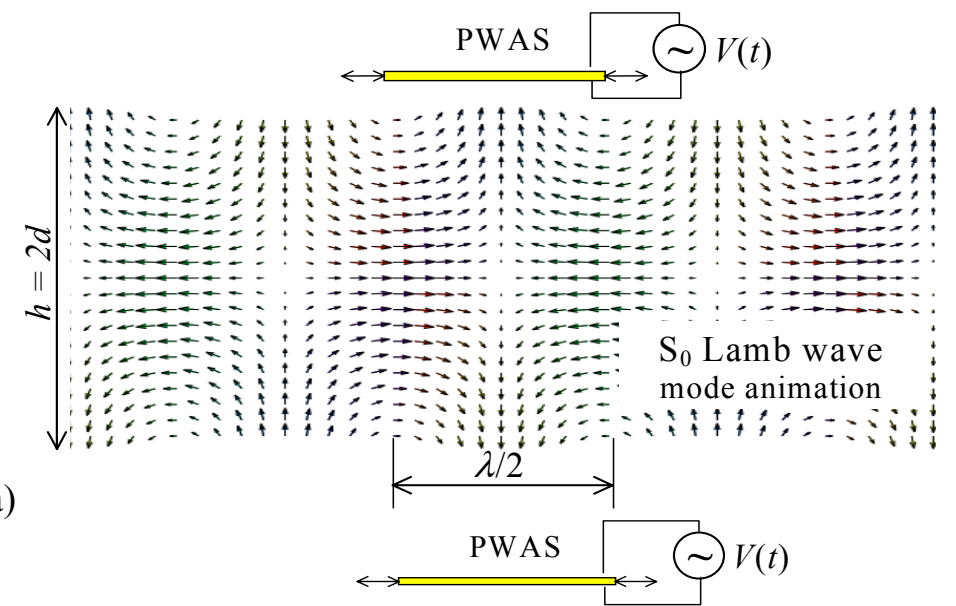

(b)

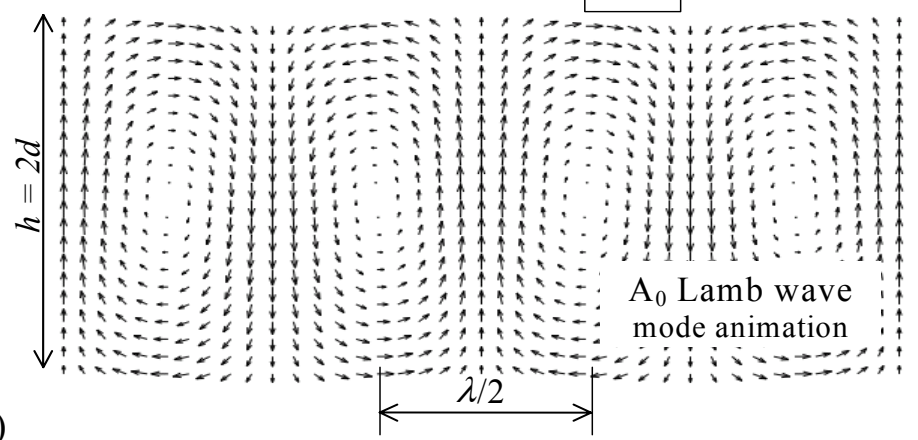

Figure 2 PWAS interaction with Lamb modes: (a) symmetric Lamb mode $\mathrm{S}_{0}$; (b) anti-symmetric Lamb mode $\mathrm{A}_{0}$

Giurgiutiu $^{15}$ studied the general Lamb-wave response of the plate under PWAS excitation using the spacedomain Fourier transforms of the basic Lamb wave equations. The strain-wave response was found in the form

$$
\varepsilon_{x}(x, t)=\frac{1}{2 \pi} \frac{-i}{2 \mu} \int_{-\infty}^{\infty}\left(\frac{\tilde{\tau} N_{S}}{D_{S}}+\frac{\tilde{\tau} N_{A}}{D_{A}}\right) e^{i(\xi x-\omega t)} d \xi
$$

where $\tilde{\tau}$ is the Fourier transform of $\tau_{a}(x), \xi$ is the wave number, $p^{2}=\frac{\omega^{2}}{c_{L}^{2}}-\xi^{2}, q^{2}=\frac{\omega^{2}}{c_{T}^{2}}-\xi^{2}$, while $c_{L}^{2}=(\lambda+2 \mu) / \rho$ and $c_{T}^{2}=\mu / \rho$ are the longitudinal (pressure) and transverse (shear) wave speeds, $\lambda$ and $\mu$ are Lame constants, $\rho$ is the mass density, $S$ and $A$ signify symmetric and antisymmetric Lamb modes, and

$$
\begin{aligned}
& N_{S}=\xi q\left(\xi^{2}+q^{2}\right) \cos p h \cos q h, \quad D_{S}=\left(\xi^{2}-q^{2}\right)^{2} \cos p h \sin q h+4 \xi^{2} p q \sin p h \cos q h \\
& N_{A}=\xi q\left(\xi^{2}+q^{2}\right) \sin p h \sin q h, \quad D_{A}=\left(\xi^{2}-q^{2}\right)^{2} \sin p h \cos q h+4 \xi^{2} p q \cos p h \sin q h
\end{aligned}
$$

A complete derivation of these expressions was given by Giurgiutiu ${ }^{16}$, pages 609-617. Note that $D_{S}$ and $D_{A}$ are the right hand sides of the Rayleigh-Lamb equations. For ideal bonding between PWAS and structure, a 
closed form solution was derived in the form:

$$
\varepsilon_{x}(x, t)=-i \frac{a \tau_{0}}{\mu} \sum_{\xi^{S}} \sin \xi^{S} a \frac{N_{S}\left(\xi^{S}\right)}{D_{S}^{\prime}\left(\xi^{S}\right)} e^{i\left(\xi^{S} x-\omega t\right)}-i \frac{a \tau_{0}}{\mu} \sum_{\xi^{A}} \sin \xi^{A} a \frac{N_{A}\left(\xi^{A}\right)}{D_{A}^{\prime}\left(\xi^{A}\right)} e^{i\left(\xi^{A} x-\omega t\right)}
$$

where $a=l_{a} / 2$ is the half-length of the PWAS and $a \tau_{0}$ is the total force transmitted by the PWAS to the structure. The summations are taken over all the symmetric $(S)$ and antisymmetric $(A)$ Lamb wave modes present in the frequency range of interest. The $\sin \xi a$ terms in Equation (3) present opportunities for Lambwave tuning. When the product $\xi a$ takes a value equal to an odd multiple of $\pi / 2$, the amplitude of $\sin \xi_{a}$ goes through a maximum, and that particular Lamb-wave mode is preferentially excited. This condition corresponds to the PWAS length, $l_{a}=2 a$, being equal to an odd multiple of the wavelength of the particular Lamb-wave mode under consideration.

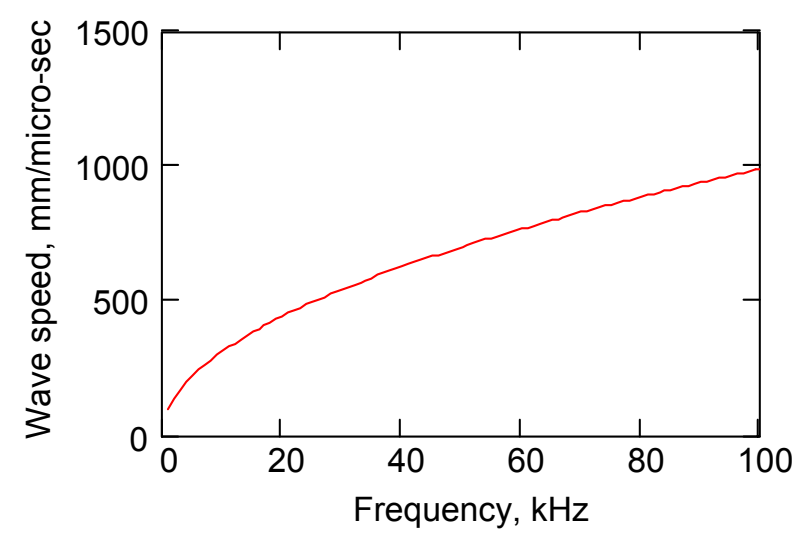

(a)

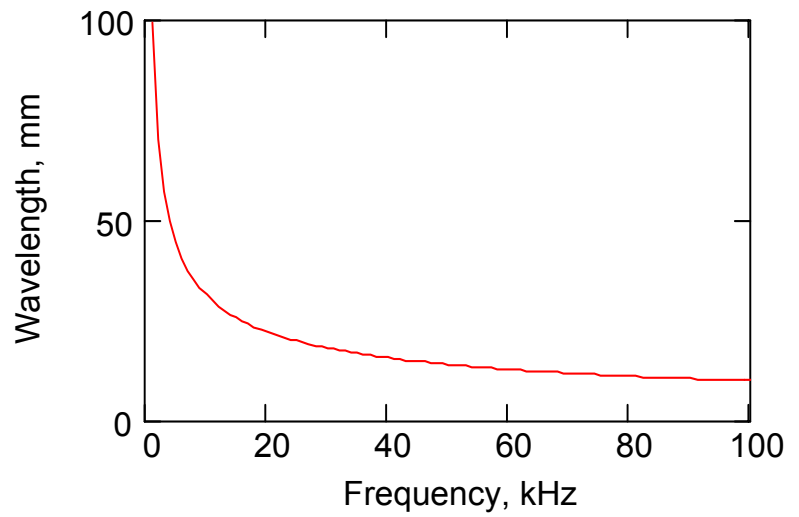

(b)

Figure 3 Dispersion characteristics of $A_{0}$ Lamb wave mode in 1-mm 2024-T3 aluminum plate: (a) $A_{0}$ wavespeed vs. frequency; (b) $A_{0}$ wavelength vs. frequency

In our analysis, we considered the excitation of the $\mathrm{A}_{0}$ Lamb wave mode which, at low frequencies, resembles flexural plate waves. The highly dispersive characteristic of $\mathrm{A}_{0}$ flexural waves makes them very sensitive to small changes in plate thickness, as expected during the corrosion process. Figure 3 shows the calculated dispersion characteristics of the $\mathrm{A}_{0}$ Lamb mode in a 1$\mathrm{mm} 2024-\mathrm{T} 3$ aluminum plate in the 1 to $100 \mathrm{kHz}$ band. It is noticed that the wavespeed and wavelength vary rapidly with frequency in this low frequency band. For tuned excitation with a $10-\mathrm{mm}$ PWAS, we are looking for wavelength of the order of $20 \mathrm{~mm}$. Figure $3 \mathrm{~b}$ indicates that a $20-\mathrm{mm}$ wavelength of the $\mathrm{A}_{0}$ mode would be encountered in the $20-40 \mathrm{kHz}$ range. A more precise determination of the PWAS tuning frequency is given in Figure 4, which shows that the 10-mm diameter PWAS would have an $\mathrm{A}_{0}$ Lamb-mode optimal tuning frequency

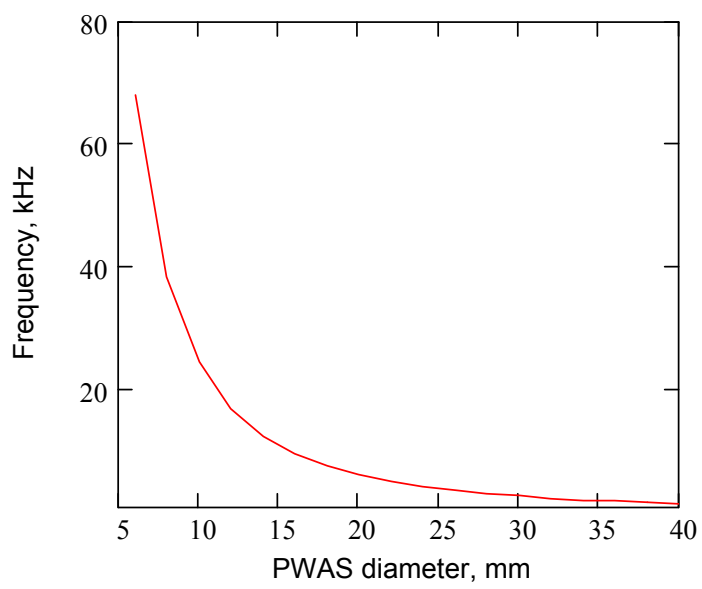

Figure 4 Variation of tuned excitation frequency with PWAS diameter for $A_{0}$ Lamb wave mode of around $30 \mathrm{kHz}$. Fine tuning experiments described in a later section of this paper have found the exact tuning frequency of $31.5 \mathrm{KHz}$. The difference between the 
theoretical prediction and experimental results is attributable to the 2-D effects, which were not captured in this 1-D analysis.

The dispersive propagation of the $\mathrm{A}_{0}$ Lamb-wave mode was analyzed using the Fourier transform method. The excitation consisted of a 3.5-count Hanning windowed smoothed tone burst of frequency $f_{0}=31.5 \mathrm{kHz}$ starting at time $t_{0}$ and lasting for the duration $T$ :

$$
p(t)=\mid \begin{array}{cc}
e^{i \omega_{0} t} \cos ^{2}\left(\pi \frac{t-t_{m}}{T}\right), \text { if } t_{0} \leq t \leq t_{0}+T \\
0 \text { otherwise }
\end{array}
$$

where $\omega_{0}=2 \pi f_{0}$. The tone burst duration was calculated in terms of the number of counts, $N_{\text {counts }}=3.5$ using the expression:

$$
T=\frac{N_{\text {counts }}}{f_{0}}
$$

The excitation was transformed to the frequency domain using the Fourier transform:

$$
\tilde{p}(f)=\int_{-\infty}^{+\infty} p(t) e^{-i \omega t} d t
$$

In the frequency domain, the excitation was multiplied with the wave transfer function (Doyle, 1997):

$$
G(k(\omega), x)=e^{-i k(\omega) \cdot x}
$$

to generate the frequency domain response:

$$
\tilde{u}(\omega, x)=p(\omega) \cdot G(\omega, x)
$$

The frequency-dependent wavenumber $k(\omega)$ of Equation (7) was calculated using the low-frequency approximation of the $\mathrm{A}_{0}$ wavespeed, i.e.,

$$
k(\omega)=\left[\frac{3 \rho\left(1-v^{2}\right)}{d^{2} E}\right]^{1 / 4} \cdot \omega^{1 / 2}
$$

Applying the inverse Fourier transform to Equation (8), we obtained the $\mathrm{A}_{0}$ mode Lamb wave response as a function of time, $t$, and location in the plate, $x$, i.e.,

$$
u(t, x)=\frac{1}{2 \pi} \int_{-\infty}^{+\infty} \tilde{u}(\omega, x) e^{i \omega t} d \omega
$$

Figure 5 presents the simulation of the $\mathrm{A}_{0}$ mode Lamb wave response at the PWAS source, $x=0$, and at distances $x=250 \sqrt{ } 2$ and $x=500 \sqrt{2} \mathrm{~mm}$ from the PWAS source. It is apparent that, as the distance from the source increases, the dispersion of the $\mathrm{A}_{0}$ mode Lamb wave packet increases. Figure 6 presents the effect of plate thickness variation on the wave packet dispersion. Under consideration is a wave packet starting at $r_{0}=$ 0 and arriving at location $r_{1}=250 \sqrt{2}$ (Figure 6a) while traveling through pristine plate, or at $r_{1 \text {-cor }}=250 \sqrt{2}$ (Figure $6 \mathrm{~b}$ ) while traveling through corroded plate that has experienced, on average, a thickness reduction of 25\%. Comparison of Figure 6a with Figure $6 \mathrm{~b}$ reveals that the effect of corrosion slows the wave packet down and to increase its dispersion. We notice that the packet traveling through the pristine region seems to reach its peak amplitude at around 280 micro-sec, while the packet traveling through 
the region with $25 \%$ average corrosion seems to reach its peak amplitude at around 320 micro-sec. This 40 micro-sec delay represents an average increase of $13 \%$ in travel time. Results similar to these predictions are to be found in the experimental section of this paper.

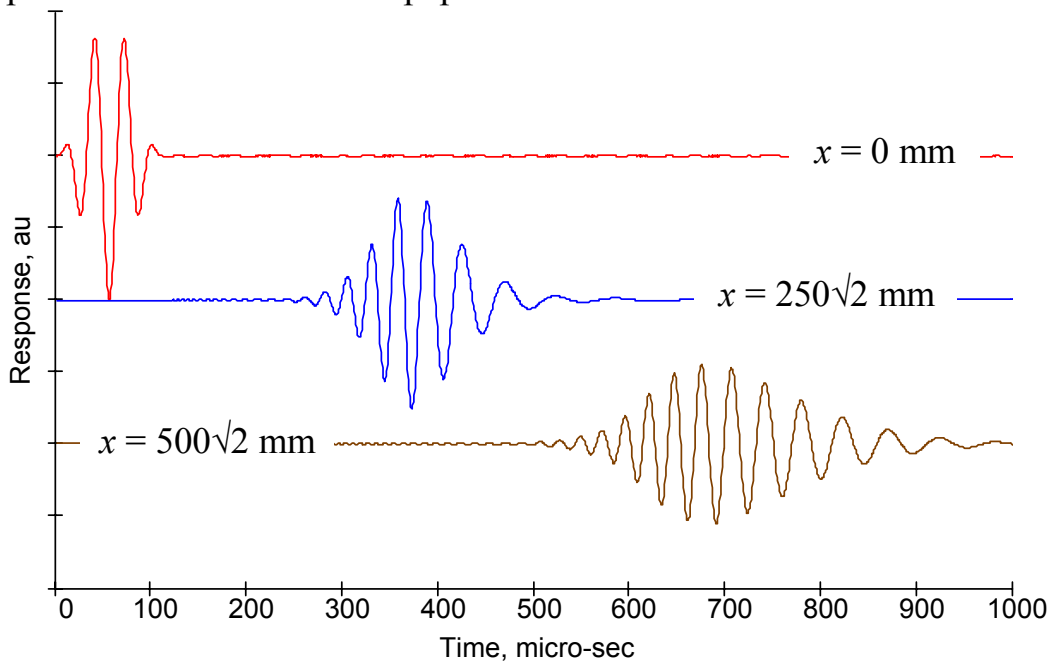

Figure 5 Simulation of $A_{0}$ mode Lamb wave response at $x=250 \sqrt{2}$ and $500 \sqrt{2} \mathrm{~mm}$ from the PWAS source placed at $x=0$ $\mathrm{mm}$ (3.5-count smoothed tone burst of $31.5 \mathrm{kHz}$, plate thickness $\mathrm{h}=1 \mathrm{~mm}$ )

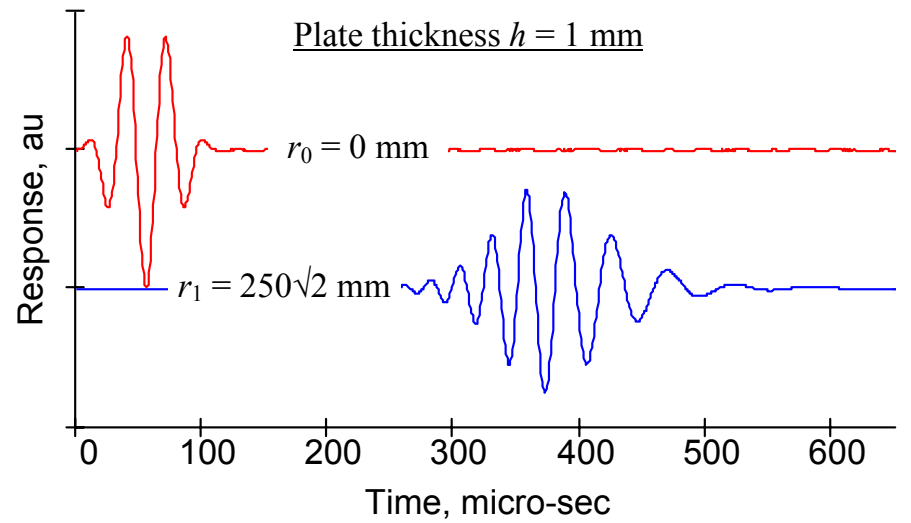

(a)

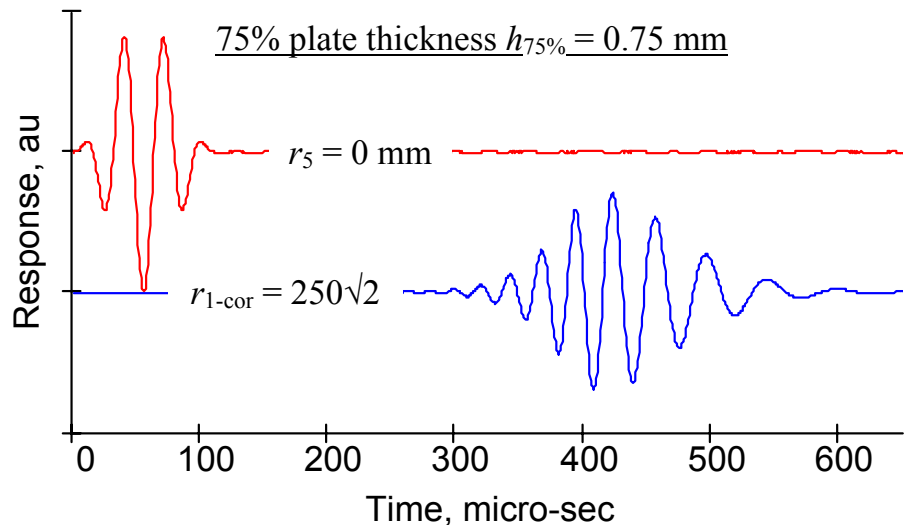

(b)

Figure 6 Simulation of $A_{0}$ Lamb wave mode response at $250 \sqrt{ } 2 \mathrm{~mm}$ from the PWAS source for two plate thickness values: (a) $\mathrm{h}=1 \mathrm{~mm} ;$ (b) $\mathrm{h}_{75 \%}=0.75 \mathrm{~mm}$ (3.5-count smoothed tone burst of $31.5 \mathrm{kHz}$ ) 
Figure 7 presents signal cross plots between the signal simulated for a pristine plate ("pristine signal") and the signal simulated for a corroded plate ("corroded signal"). These plots are very sensitive to small shifts in signal phase. Two charts are presented, one for $0.5 \%$ total material loss (Figure $7 \mathrm{a}$ ) and one for $1.0 \%$ total material loss (Figure 7b). In each chart, two plots are made: first, a plot of the pristine signal on itself, which results in a straight $45^{\circ}$ line; second, a plot of the corroded signal against the pristine signal. This second plot results in an ellipse. By comparing Figure $7 \mathrm{a}$ with Figure $7 \mathrm{~b}$ we observe that the width of the ellipse correlates with the simulated corrosion intensity. For mild corrosion ( $0.5 \%$ total material loss $)$, the ellipse is relatively slim (Figure $7 \mathrm{a})$. For more intense corrosion, (1.0\% total material loss) the ellipse is much wider. These results indicate that the signal cross plots can be effectively used to identify the signal phase shifts and dispersions due to the presence of corrosion.

\section{$\underline{0.5 \% \text { total material loss }}$}

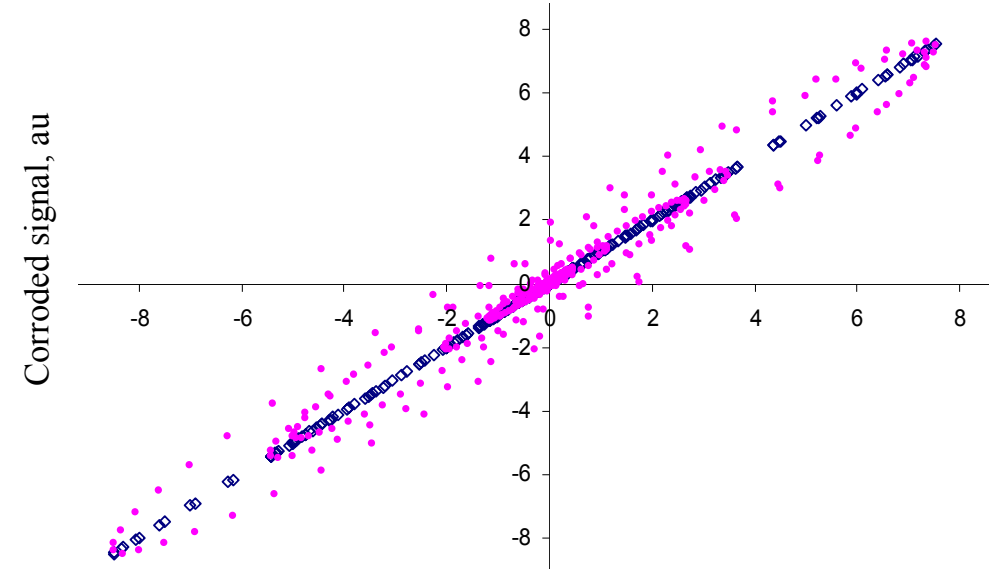

(a)

Pristine signal, au

\section{$1.0 \%$ total material loss}

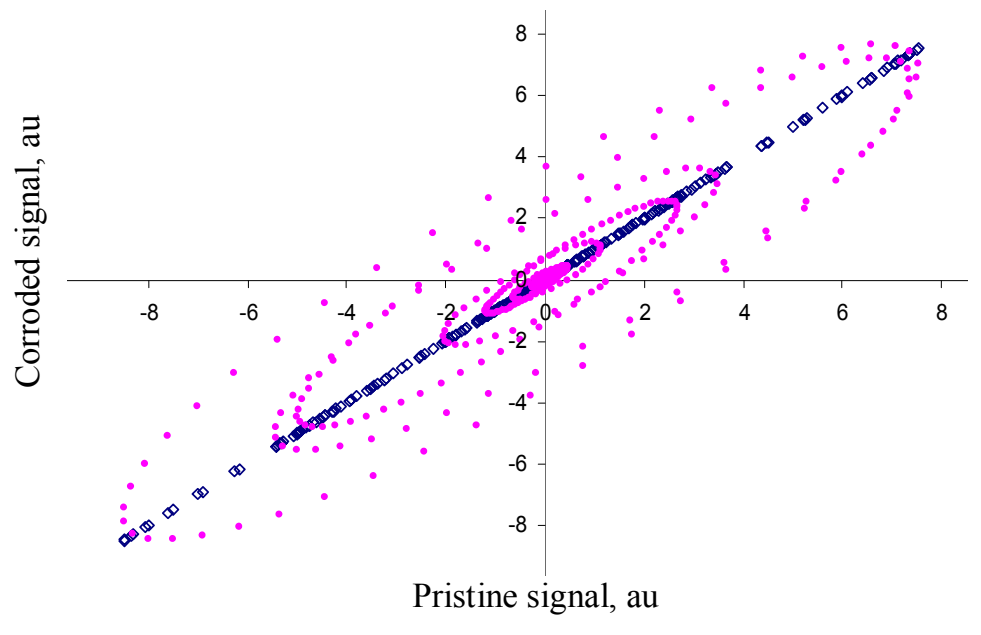

Figure 7 Cross plot between the pristine and corroded signals: (a) $0.5 \%$ total material loss; (b) $1 \%$ total material loss 


\section{EXPERIMENTAL SETUP}

The use of PWAS generated antisymmetric Lamb waves to detect material loss was demonstrated by laboratory experiments. A plate of aluminum alloy 2024 that was $750 \mathrm{~mm} \mathrm{x} 750 \mathrm{~mm} \mathrm{x} 1 \mathrm{~mm}$ was instrumented with two 10-mm diameter 0.2-mm thick PWAS (American Piezo Ceramics, APC-850) along the diagonal line of the square plate. The PWAS were positioned $25.0 \mathrm{~cm}$ apart (nearest point to nearest point), and at least $29.3 \mathrm{~cm}$ from the nearest edge of the plate. A 3.5 count smoothed tone burst was generated by a HP33120A function generator which propagated as an $\mathrm{A}_{0}$ Lamb wave and was detected by a LeCroy LT584 DSO digital oscilloscope (Figure 8).

Table 1 Size and depth of damage on the Al-2024 plates

\begin{tabular}{ccc}
\hline Plate \# & Diameter of Corroded Area & Depth of Corroded Area \\
\hline $\mathbf{9}$ & $15 \mathrm{~mm}$ & $0.26 \mathrm{~mm}$ \\
\hline $\mathbf{7}$ & $15 \mathrm{~mm}$ & $0.14 \mathrm{~mm}$ \\
\hline
\end{tabular}

The simulated corrosion damage was created on the aluminum plates by using hydrochloric acid, which was confined to a particular area of the center of the plate. The acid was confined to a particular area of the plate using Tolber Microstop Stop-Off Lacquer to adhere a rubber washer with an inner diameter of the desired diameter of the corrosion area to the aluminum plate. Depth of the corrosion area was controlled by time. The longer the acid was used on the plate the deeper the corroded area. Two different plates were corroded. The dimensions and depths of the damage are in Table 1 below. For the 0.26-mm thickness loss, the acid was used for 80 minutes, and it was refreshed every 10 minutes by removing the acid present and replacing it with fresh acid. When the desired depth had been reached, the acid was neutralized with sodium bicarbonate solution. These measurements are only approximate since like real corrosion the edges are not perfectly round and there are many pits and irregularities in the simulated corrosion. After the plate was corroded, the PWAS were connected again to the function generator and the oscilloscope for data collection. These traces were then compared to the undamaged ones to detect damage.

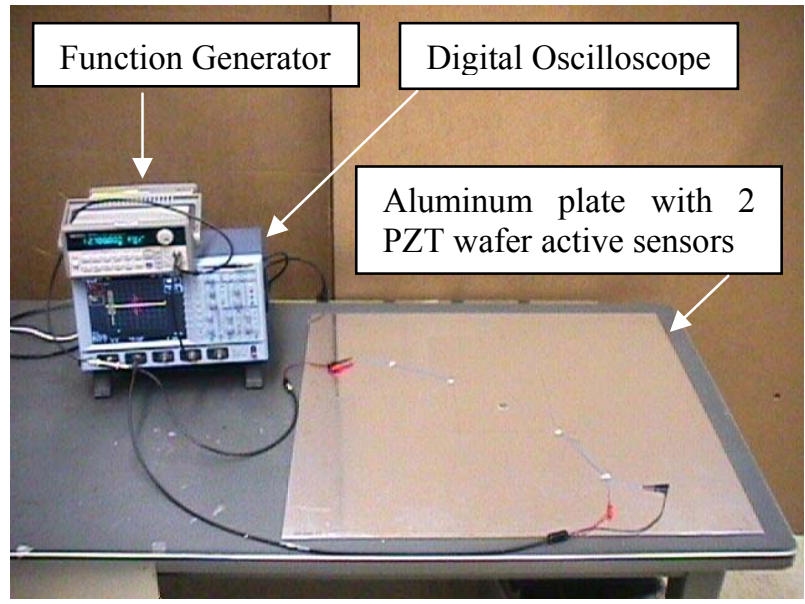

(a)

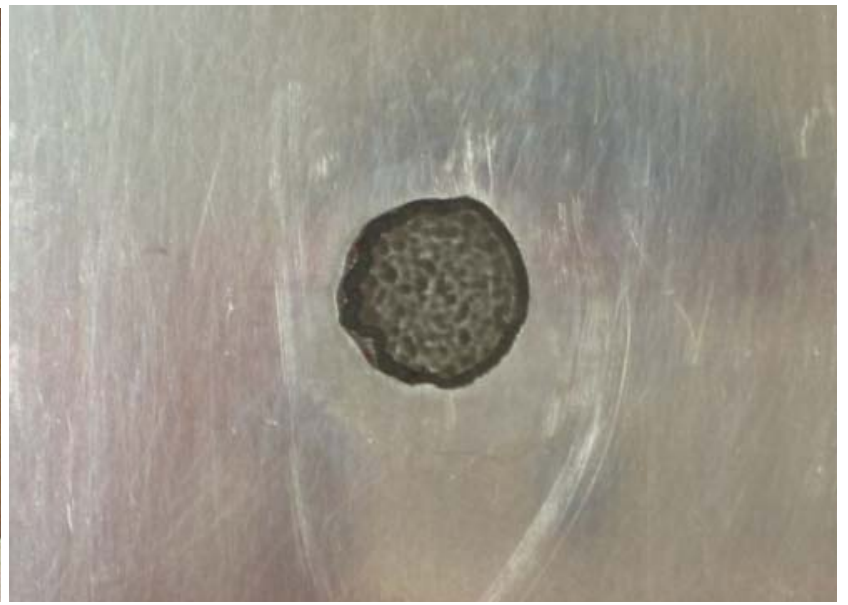

(b)

Figure 8 Experimental setup for the square plate material loss detection experiment: (a) overall view showing the oscilloscope, function generator and instrumented plate; (b) detail of the topography of one of the areas of simulated corrosion. 


\section{RESULTS}

Figure 9 presents data acquired from plate 7 before and after it is damaged. As expected from the theoretical analysis, the damaged signal has a greater arrival time and is more dispersed. These are measurable, but they are very subtle and might be within the noise range. However, two other effects are easier to observe. With the presence of damage, the $\mathrm{A}_{0}$ wave packet increases in amplitude and there is a significant measurable phase shift.

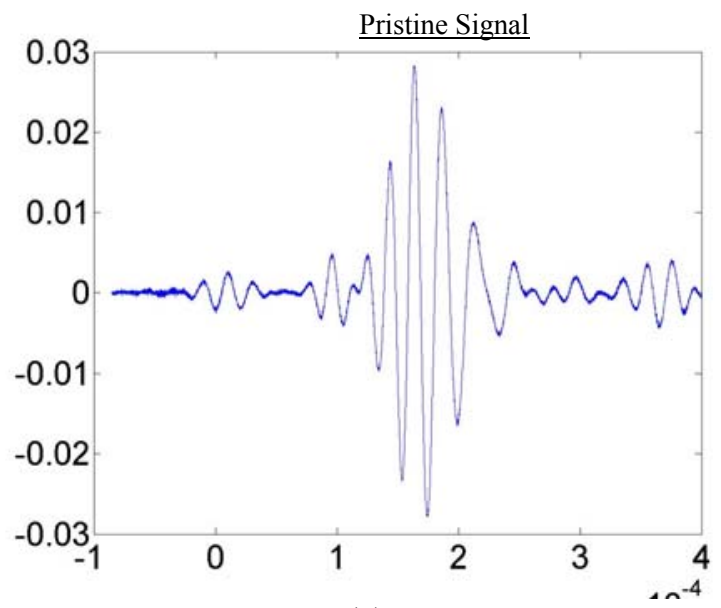

(a)

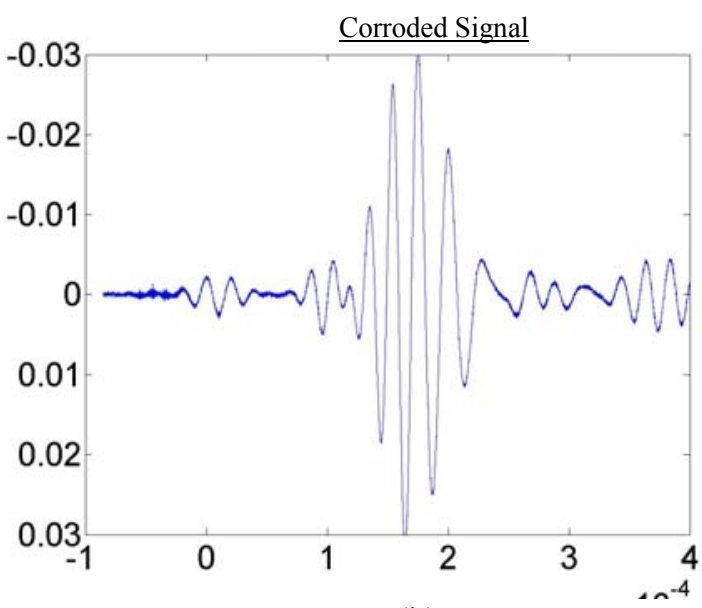

(b)

Figure 9 Cross plot between the pristine and corroded signals, amplitude in Volts on the $y$-axis and time in seconds on the $x$ axis: (a)Pristine; (b) Corroded Signal

Figure 10 shows pristine and corroded signals from plate 9. Plate 9 has nearly twice as much thickness loss as plate 7. As expected, plate 9 had a greater $A_{0}$ amplitude increase than plate 7 when it was damaged. However, there is not as large amount of a change in the arrival time or dispersion.

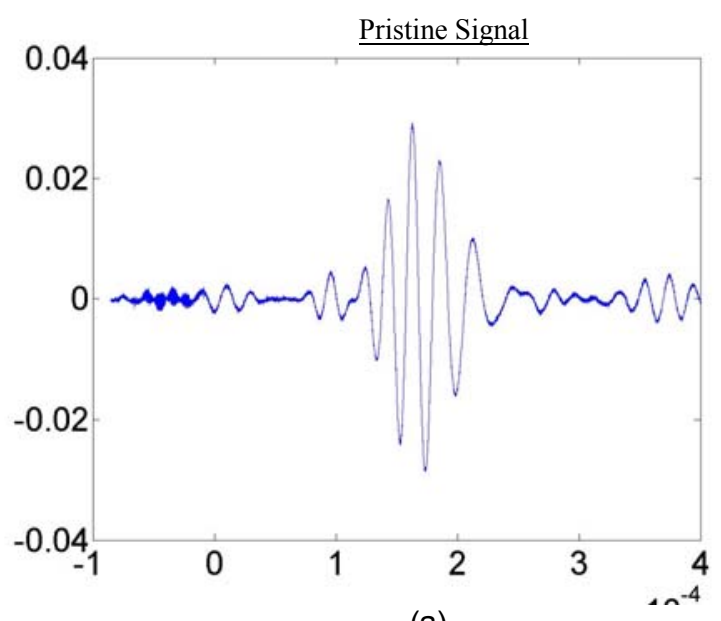

(a)

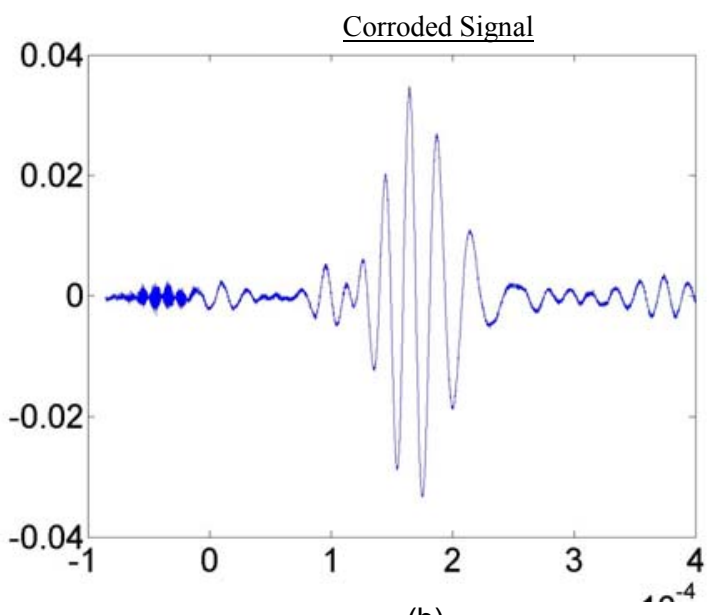

(b)

Figure 10 Cross plot between the pristine and corroded signals, amplitude in Volts on the $y$-axis and time in seconds on the $x$ axis: (a) Pristine Signal; (b) Corroded Signal 


\section{DATA PROCESSING AND INTERPRETATION}

To observe the amplitudes for the $\mathrm{A}_{0}$ mode, the envelope of the signal was taken. (Figure 11) To find the envelope of a signal, a Hilbert transform was used on the data. In comparing the amplitudes of the pristine envelope to the plate 7 signal envelope and the plate 9 signal envelope the data suggests a linear relationship. Further analysis is needed to confirm this.

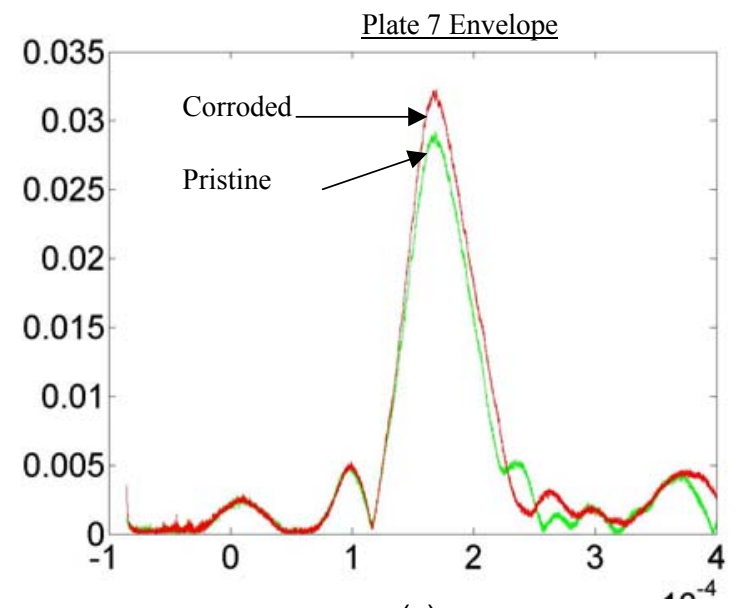

(a)

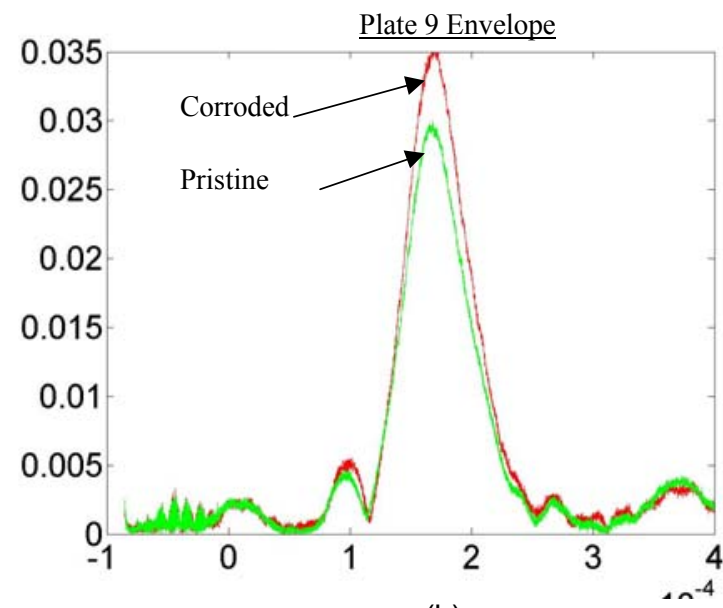

(b)

Figure 11 Envelope plot of the pristine and corroded signals, amplitude in Volts on the $y$-axis and time in seconds on the $x$ axis: (a) Plate 7 with $0.14 \mathrm{~mm}$ thickness loss; (b) Plate 9 with $0.26 \mathrm{~mm}$ thickness loss

In order to examine the phase shift, we constructed cross plots between the corroded and the pristine signals, as described in the theory section of this paper. As the theory suggests, the ellipse widens with increased corrosion damage. (Figure 12)

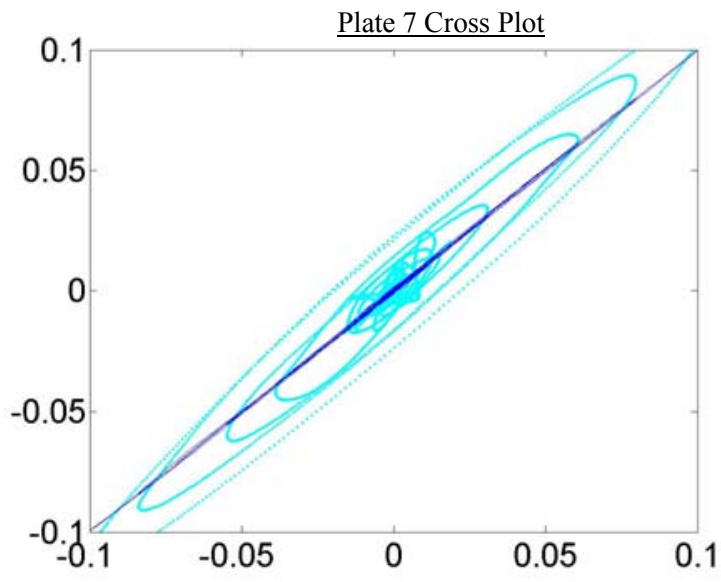

(a)

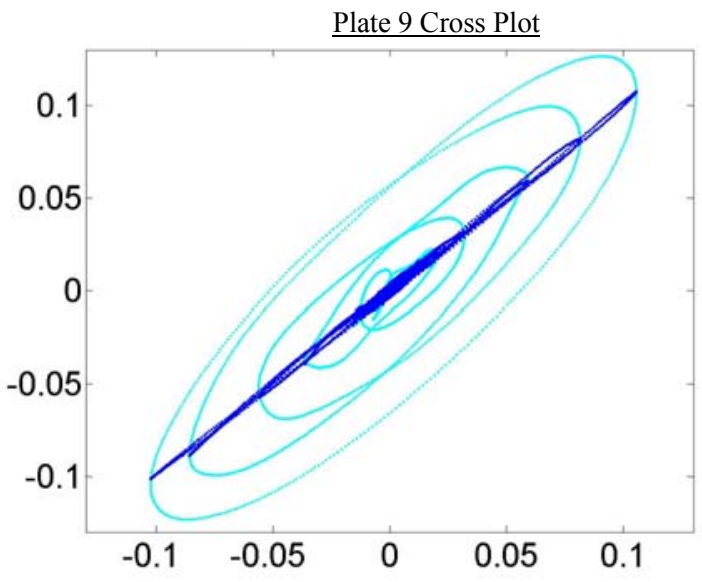

(b)

Figure 12 Cross plot between the pristine and corroded signals, amplitude in Volts on the x-axis and y-axis: (a) Plate 7 with $0.14 \mathrm{~mm}$ thickness loss; (b) Plate 9 with $0.26 \mathrm{~mm}$ thickness loss 


\section{SUMMARY AND CONCLUSIONS}

A method for in-situ corrosion detection using piezoelectric wafer active sensors (PWAS) permanently attached to the structure is presented. The method is based on flexural ultrasonic guided waves $\left(\mathrm{A}_{0}\right.$ Lamb mode) transmitted by one PWAS and received by the other PWAS in pitch-catch mode. Theoretical predictions were validated by experimental results. We were able to detect minute changes in arrival time due to simulated corrosion damage and we could detect a small amount of increased dispersion. However, these effects were very small. In addition, we were better able to observe an amplitude increase by analyzing the waveform envelope. We could also detect a considerable phase change by analyzing a cross plot between the pristine signal and the corroded signal. All four of these effects seem to have a linear relationship. In the future, we plan to continue our studies in order to actually quantify damage based upon the measurement of these effects for different corrosion depth and intensity.

\section{ACKNOWLEDGMENTS}

The financial support of AFRL/ML in conducting this research is thankfully acknowledged. We would like to acknowledge the personal contributions of John Barnes and Dr. Shamachary Sathish for their advice and counsel, and of Chris Kacmar for his assistance in creating the corrosion samples.

\section{REFERENCES}

1. Dalton, R. P.; Cawley, P.; Lowe, M. J. S. (2001) "The Potential of Guided Waves for Monitoring Large Areas of Metallic Aircraft Structure", Journal of Nondestructive Evaluation, Vol. 20, pp. 29-46, 2001

2. Rose, J. L. (2002) "A Baseline and Vision of Ultrasonic Guided Wave Inspection Potential”, ASME Journal of Pressure Vessel Technology, Vol. 124, No. 3, August 2002, pp. 273-282

3. Alleyne, D. N.; Cawley, P. (1992) "The Interaction of Lamb Waves with Defects", IEEE Transactions on Ultrasonics, Ferroelectrics, and Frequency Control, Vol. 39, No. 2, May 1992, pp. 381-397

4. Chona, R.; Suh, C. S.; Rabroker, G. A. (2003) "Characterizing Defects in Multi-Layer Materials Using Guided Ultrasonic Waves", Optics and Lasers in Engineering, Vol. 40 (2003), pp. 371-378

5. Chahbaz, A.; Mustafa, V.; Hay, D. (1996) "Corrosion Detection in Aircraft Structures Using Guided Lamb Waves" ASNT 1996 Fall Conference, Seattle, WA, http://www.ndt.net/article/tektrend/tektrend.htm

6. Alleyne, D.N., Pavlakovic, B., Lowe, M.J.S., Cawley, P. (2001) "Rapid, Long Range Inspection of Chemical Plant Pipework using Guided Waves," Review of Progress in QNDE, Vol. 20, (2001), pp. 180-187

7. Jenot, F.; Ouaftouh, M.; Duquennoy, M.; Ourak, M. (2001) "Corrosion Thickness Gauging in Plates Using Lamb Wave Group Velocity Measurements” Measurement Science and Technology, IOP Publishing Ltd., UK, vol. 12, 2001, p12871293

8. Sicard, R.; Chahbaz, A.; Goyette, J. (2003) "Corrosion Monitoring of Airframe Structures Using Ultrasonic Arrays and Guided Waves” AIP conference Proceedings, AIP, US, no. 657A, 2003, p806-813

9. Keilers, C.H.; Chang, F.-K. (1995) "Identifying Delamination in Composite Beam Using Built-in Piezoelectrics" Journal of Intelligent Material Systems and Structures, Vol. 6, 1995, p647-672

10. Wang, C.S.; Chang, F.-K. (2000) "Built-in Diagnostics for Impact Damage Identification of Composite Structures" Proceedings of the $2^{\text {nd }}$ International Workshop on Structural Health Monitoring, 8-10 September 1999, Stanford, CA, p612-621

11. Kessler, S.; Spearing, s. M.; Soutis, C. (2002) "Damage Detection in Composite Materials Using Lamb Wave Methods" Smart Materials and Structures, IOP Publishing Ltd., UK, vol. 11, 2002, p269-278

12. Lemistre, M.; Osmont, D.; Balageas, D. (2000) "Active Health System Based on Wavelet Transform Analysis of Diffracted Lamb Waves", 5th European Conf. on Smart Structures and Materials, SPIE Vol. 4073 (2000), pp. 194-202

13. Giurgiutiu, V.; Zagrai, A. N.; Bao, J. (2002) "Piezoelectric Wafer Embedded Active Sensors for Aging Aircraft Structural Health Monitoring”, Structural Health Monitoring - An Int. Journal, Sage Pub., Vol. 1, No. 1, July 2002, pp. 41-61

14. Giurgiutiu, V.; Bao, J. (2002) "Embedded-Ultrasonics Structural Radar for the Nondestructive Evaluation of Thin-Wall Structures" Proceedings of the 2002 ASME International Mechanical Engineering Congress, November 17-22, 2002, New Orleans, LA, paper \# IMECE2002-39017

15. Giurgiutiu, V. (2003) "Lamb Wave Generation with Piezoelectric Wafer Active Sensors for Structural Health Monitoring", SPIE's $10^{\text {th }}$ International Symposium on Smart Structures and Materials and $8^{\text {th }}$ Annual International Symposium on NDE for Health Monitoring and Diagnostics, 2-6 March 2003, San Diego, CA, paper \# 5056-17

16. Giurgiutiu, V.; Lyshevski, S. E. (2004) Micro Mechatronics: Modeling, Analysis, and Design with MATLAB, CRC Press, ISBN 084931593X, 2004 